\title{
Some Properties of Soft Categories
}

\author{
Simge Öztunç
}

\begin{abstract}
In this paper we recalled some definitions and properties of soft set theory and soft category theory. We investigated new properties of soft category theory such asconcept of initial and terminal objects. Also we give the definition of soft connected category and we obtain the necessary and sufficient condition to have a zero object for soft category.
\end{abstract}

Index Terms-Soft category, initial object, terminal object, connected soft category.

\section{INTRODUCTION}

The concept of soft sets was introduced by D. Molodtsov [1] in 1999 and Soft set theory became an alternative and useful tool for computer science, modeling problems in engineering, economics, medical and social science.

Theorical properties of soft set theory has also been studied some mathematicians. Maji et al. [2] introduced several operations on soft sets. Aktaş and Çağman [3] defined the notion of soft groups and developed the algebraic properties of soft groups. Some conceps in soft topological spaces studied by Zorlutuna et al. [4] Also Aras, Sönmez, Çakallı [5] and Zorlutuna and Çakır [6] worked on continuity of soft mappings.

On the other hand Category Theory was studied by many mathematicians. Eilenberg and Mac Lane's 'General Theory of Naturel Equivalences' [7] was the first original paper in which the theory was formulated. Category Theory has an important place, because it plays multidisciplinary roles amoung many branches of mathematics. Also Category Theory may be consideder as study of (abstract) algebras of functions.

The soft category theory studied by Sardar and Gupta in [8]. They introduced the basic notions of the theory of soft categories and gave some introductory result of the soft category theory.

The purpose of this paper is to study some new properties of soft category theory. Sardar and Gupta defined in [8] the initial and terminal objects and zero objects in soft categorie and we show that objects of the same type in a soft category are isomorphic. Also we defined connected soft category and gave the necessary and sufficient condition to be connected soft category.

Manuscript received April 24, 2015; revised April 23, 2016. This Paper is supported by Celal Bayar University Scientific Research Project Coordination Unit (CBU BAP- Project No:2015-109).

Simge Öztunç is with the Celal Bayar University, Faculty of Science and Arts, Department of Mathematics Muradiye Campus, 45047, Manisa, Turkey (e-mail: simge.oztunc@cbu.edu.tr).

\section{PRELEMINARIES}

In this section we give some definitions of Soft Set Theory an Category Theory.

Definition 2.1. [2] A pair $(F, A)$ is called a soft set over the universe $X$, where $F$ is a mapping given by

$$
F: A \rightarrow P(X)
$$

and $A \subseteq E$.

Any soft set $(F, A)$ can be extended to a soft set of type $(F, E)$, where

$$
F(e) \neq \varnothing
$$

for all $e \in A$ and

$$
F(e)=\varnothing
$$

for all $e \in E \backslash A . S(X, E)$ denotes the family of all soft sets over $X$ [2].

Definition 2.2. [2, 9] Let $(F, A),(G, B) \in S(X, E)$.

1) $(F, A)$ is a soft subset of $(G, B)$, denoted by $(F, A) \subseteq \tau(G, B)$,

if $F(e) \subseteq G(e)$ for each $e \in E$.

2) $(F, A)$ and $(G, B)$ are said to be soft equal, denoted by

$$
(F, A)=(G, B)
$$

if $(F, A) \tilde{\subseteq}(G, B)$ and $(G, B) \widetilde{\subseteq}(F, A)$.

3) Union of $(F, A)$ and $(G, B)$ is a soft set $(H, C)$, where

$$
C=A \cup B \text { and } H(e)=F(e) \cup G(e)
$$

for each $e \in E$. This is denoted by

$$
(F, A) \sim(G, B)=(H, C)
$$

4) Intersection of $(F, A)$ and $(G, B)$ is a soft set $(H, C)$,

where

$$
C=A \cap B \text { and } H(e)=F(e) \cap G(e)
$$


for each $e \in E$. This relationship is written as

$$
(F, A) \tilde{\cap}(G, B)=(H, C) .
$$

Definition 2.3. [2], [9] Let $(F, A) \in S(X, E) .(F, A)$ is said to be a null soft set, denoted by $\Phi$, if $\forall e \in E, F(e)=\varnothing$.

Definition 2.4. [2] A soft set $(F, A)$ over $X$ is said to be an absolute soft set, denoted by $\tilde{A}$ if for all $a \in A, F(a)=X$.

Definition 2.5 [10] A category $\mathcal{C}$ consists of the following data:

- Objects: $A, B, C, \ldots$

- Arrows: $f, g, h, \ldots$

- For each arrow $f$, there are given objects

$\operatorname{dom}(f), \operatorname{cod}(f)$

called the domain and codomain of $\quad$ fWe write

$$
f: A \rightarrow B
$$

To indicate that

$$
A=\operatorname{dom}(f) \text { and } B=\operatorname{cod}(f)
$$

Given arrows $f: A \rightarrow B$ and $g: B \rightarrow C$, that is, with

$$
\operatorname{cod}(f)=\operatorname{dom}(g)
$$

there is given an arrow

$$
g \circ f: A \rightarrow C
$$

called the composite of $f$ and $g$.

For each object $A$, there is given an arrow

$$
1_{A}: A \rightarrow A
$$

called the identity arrow of $A$.

This data required to satisfy the following laws:

Associativity :

$$
h \circ(g \circ f)=(h \circ g) \circ f
$$

for all $f: A \rightarrow B, g: B \rightarrow C$ and $h: C \rightarrow D$.

Unit :

$$
f \circ 1_{A}=f=1_{B} \circ f
$$

for all $: f \quad A \quad B$

Definition 2.6. [10] In any category $\mathcal{C}$, an arrow $f: A \rightarrow B$ is called an monomorphism, if given any $g, h: C \rightarrow A, f \circ g=f \circ h$ implies $g=h$,

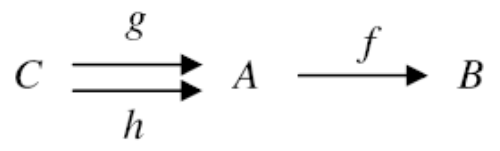

Definition 2.7. [10] In any category $\mathcal{C}$, an arrow $f: A \rightarrow B$ is called an monomorphism, if given any $i, j: B \rightarrow D, i \circ f=j \circ f$ implies $i=j$,

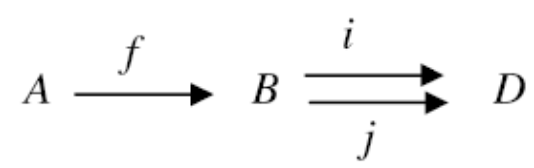

Definition 2.8. [10] In any category $\mathcal{C}$, an arrow $f: A \rightarrow B$ is called an isomorphism, if there is an arrow

$$
g: B \rightarrow A
$$

in $\mathcal{C}$ such that

$$
g \circ f=1_{A} \text { and } f \circ g=1_{B} .
$$

Definition 2.9. [10] A functor

$$
F: \mathcal{C} \rightarrow \mathcal{D}
$$

Between $\mathcal{C}$ and $\mathcal{D}$ is a mapping of objects to objects and arrows to arrows in such a way that

- $F(f: A \rightarrow B): F(f): F(A) \rightarrow F(B)$

- $F\left(1_{A}\right): 1_{F(A)}$

- $F(g \circ f)=F(g) \circ F(f)$.

That is $F$ preserves domains and codomains, identity arrows, and composition. A functor

$$
F: \mathcal{C} \rightarrow \mathcal{D}
$$

Thus gives a sort of picture of $\mathcal{C}$ in $\mathcal{D}$.

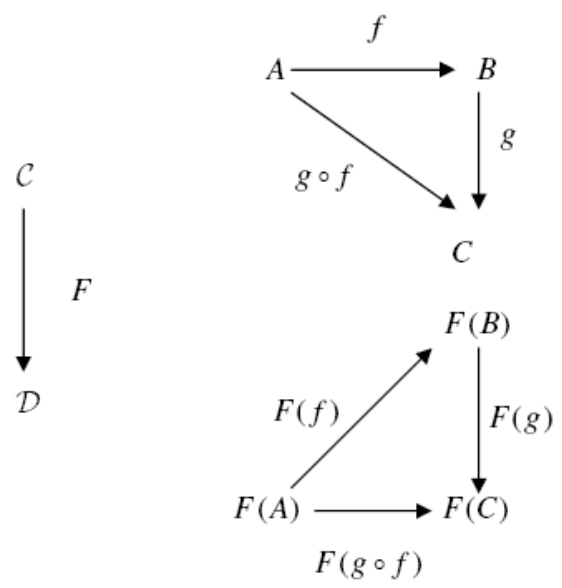


Definition 2.10. [11] An object $U$ of a category $\mathcal{C}$ is said to be an initial object if for every object $X$ of $\mathcal{C}$, the set $\operatorname{Mor}_{\mathcal{C}}(U, X)$ is a singeleton. Dually $U$ is said to be a terminal object if for every object $X$ of $\mathcal{C}$, the set

$$
\operatorname{Mor}_{\mathcal{C}}(X, U)
$$

is a singeleton

\section{Some Properties OF SOFt CATEgories}

Definition 3.1. [8] Let $\mathcal{C}$ be a category, $\mathcal{P}(\mathcal{C})$ is the set of all subcategories of $\mathcal{C}$ and $A$ a set of parameters. Let $F: A \rightarrow \mathcal{P}(\mathcal{C})$ be a mapping. Then $(F, A)$ is said to be a soft category over $\mathcal{C}$ if $F(x)$ is a subcategory of $\mathcal{C}$.

Definition 3.2. [8] Let $(F, A)$ be a soft category over $\mathcal{C}$. Then

$$
(F, A)
$$

is said to be a soft category with initial objecets over $\mathcal{C}$ if $F(x)$, as a category has initial objects for all $x \in A$.

Definition 3.3. [8] Let $(F, A)$ be a soft category over $\mathcal{C}$. Then

$$
(F, A)
$$

is said to be a soft category with terminal objecets over $\mathcal{C}$ if $F(x)$, as a category has terminal objects for all $x \in A$.

Theorem 3.4. Let $(F, A)$ be a soft category over $\mathcal{C}$. Then any two initial objects in a soft category $(F, A)$ are isomorphic.

Proof: Assume that $(F, A)$ is a soft category over $\mathcal{C}$. Let $U_{1}$ and $U_{2}$ be initial objects in a soft category $(F, A)$. Then $U_{1}$ and $U_{2}$ are initial objects in $F(x)$. We must show contains an isomorphism. If $U_{1}$ is an initial object for $F(x)$, then

$$
\begin{gathered}
\operatorname{Mor}_{F(x)}\left(U_{1}, U_{2}\right) \\
\operatorname{Mor}_{F(x)}\left(U_{1}, U_{2}\right)=\{\alpha\}
\end{gathered}
$$

For any object $U_{2}$. If $U_{2}$ is an initial object for $F(x)$, then

$$
\operatorname{Mor}_{F(x)}\left(U_{2}, U_{1}\right)=\{\beta\}
$$

For any object $U_{1}$. We get

$$
\alpha \circ \beta \in \operatorname{Mor}_{F(x)}\left(U_{2}, U_{2}\right)
$$

By the composition property of morphisms. Therefore $\alpha \circ \beta=i d_{U_{2}}$.

Similarly we have

$$
\beta \circ \alpha=i d_{U_{1}} .
$$

It follows that $\alpha$ is an isomorphism with

$$
\beta=\alpha^{-1}
$$

and $U_{1}$ and $U_{2}$ are isomorphic.

Theorem 3.5. Let $(F, A)$ be a soft category over $\mathcal{C}$ and $f \in \operatorname{Mor}_{F(x)}(X, A)$. If $X$ is a terminal object in a soft category $(F, A)$. Then, $f$ is monomorphism.

Proof: Let $(F, A)$ be a soft category over $\mathcal{C}$. Assume that $X$ is a terminal object and in $F(x)$ and $h, k \in \operatorname{Mor}_{F(x)}(C, X)$. Let $f \circ h=f \circ k$. Consider the following diagram

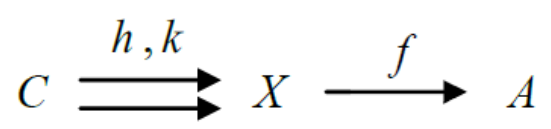

We obtained $h=k$, since $X$ is a terminal object in $F(x)$. Hence $f$ is a monomorphism.

Theorem 3.6. Let $(F, A)$ be a soft category over $\mathcal{C}$ Then any two terminal objects in a soft category $(F, A)$ are isomorphic.

Proof: Let $(F, A)$ be a soft category over $\mathcal{C}$. Assume that $O_{1}$ and $O_{2}$ are terminal objects in a soft category $(F, A)$. Then $O_{1}$ and $O_{2}$ are terminal objects in $F(x)$. We have to show that

$$
\operatorname{Mor}_{F(x)}\left(O_{1}, O_{2}\right)
$$

Contains an isomorphism. If $O_{1}$ is a terminal object for $F(x)$, then

$$
\operatorname{Mor}_{F(x)}\left(O_{2}, O_{1}\right)=\{f\}
$$

For any object $O_{2}$. If $O_{2}$ is a terminal object for $F(x)$, then

$$
\operatorname{Mor}_{F(x)}\left(O_{1}, O_{2}\right)=\{g\}
$$

For any object $O_{1}$. We have 


$$
g \circ f=i d_{O_{1}}
$$

And similarly

$$
f \circ g=i d_{O_{2}}
$$

By the composition property of morphisms. Therefore $f$ is an isomorphism with

$$
f=g^{-1}
$$

And $\mathrm{O}_{1}$ and $\mathrm{O}_{2}$ are isomorphic. Corollary 3.7. The objects of the same type in a soft category are isomorphic.

Proof: The proof is obtained by Theorem 3.5 and Theorem 3.6.

Definition 3.8. [8] Let $(F, A)$ be a soft category over $\mathcal{C}$. Then

$$
(F, A)
$$

Is said to be a soft category with zero objects over $\mathcal{C}$ if $F(x)$, as a category has zero object.

Definition 3.9. A soft category over $\mathcal{C}$ is said to be connected if

$$
\operatorname{Mor}_{F(x)}(A, B) \neq \varnothing
$$

for all objects $A, B$ of $(F, A)$.

Theorem 3.10. Let $(F, A)$ be a soft category over a category $\mathcal{C}$ with an initial object and a terminal object. Then $(F, A)$ has a zero object if and only if $(F, A)$ is connected.

Proof: If $Z$ is a zero object for $(F, A)$. Hence $Z$ a zero object for $F(x)$. Then for every morphism $\alpha$, we have

$$
\begin{aligned}
X \stackrel{\alpha}{\longrightarrow} A \stackrel{0_{A B}}{\longrightarrow} B & =X \stackrel{\alpha}{\longrightarrow} A \stackrel{0_{A Z}}{\longrightarrow} Z \stackrel{0_{Z B}}{\longrightarrow} B \\
& =X \stackrel{0_{X Z}}{\longrightarrow} Z \stackrel{0_{Z B}}{\longrightarrow} B \\
& =X \stackrel{0_{X B}}{\longrightarrow} B
\end{aligned}
$$

For any object $X, A, B$ of soft category $(F, A)$. Writing all zero morphisms as 0 , It follows that $0 \circ \alpha=0$ and similarly we have $\alpha \circ 0=0$. Hence it is obtained that $(F, A)$ is connected.

Conversely suppose that $(F, A)$ is connected. Let $X$ be an initial object and $Y$ is a terminal object for $F(x)$. We observe that if

$$
\operatorname{Mor}_{F(x)}(X, Y)=\{\alpha\} \neq \varnothing,
$$

Then $\alpha$ is monic. In fact since $(F, A)$ is connected. There exists

$$
\beta: Y \rightarrow X
$$

and we have

$$
\beta \circ \alpha=i d_{X}
$$

Since $X$ is initial. Thus $\alpha$ is a section and hence is monic. Assume that

$$
f, g \in \operatorname{Mor}_{F(x)}(A, X) \text {. }
$$

Then

$$
\alpha \circ f, \alpha \circ g \in \operatorname{Mor}_{F(x)}(A, Y)
$$

From the composition property. We get

$$
\alpha \circ f=\alpha \circ g
$$

Since $\alpha$ is monic, it is obtained that

$$
f=g
$$

Consequently we see that $X$ is also terminal object for $F(x)$, hence is a zero object.

\section{CONCLUSION}

We have worked on some properties of soft categories in this paper. We concluded that the objects of the same type in a soft category are isomorphic. Also we defined the connectedness of a soft category and consequently obtained the sufficient and necessary condition to be soft connected.

\section{ACKNOWLEDGMENT}

Author(s) thanks to Celal Bayar University Scientific Research Project Coordination Unit for their financial support.

\section{REFERENCES}

[1] D. Molodtsov, "Soft set theory-first result," Comput. Math, Appl., vol 37, pp. 19-31, 1999

[2] P. K. Maji, R. Biswas, and A. R. Roy, "Soft set theory," Comput. Math, Appl., vol. 45, pp. 555-562, 2003.

[3] H. Aktas and N. Cagman, "Soft sets and soft groups," Inform. Sci., no. 177, pp. 2726-2735, 2007.

[4] İ. Zorlutuna, M. Akdağ, W. K. Min, and S. Atmaca, "Remarks on soft topological spaces," Ann. Fuzzy Math. Inform, no. 3, pp. 171-185, 2011.

[5] Ç. G. Aras, A. Sönmez, and H. Çakallı, "On soft mappings, arxiv computers and mathematics with applications," vol. 60, no. 9, 2013.

[6] İ. Zorlutunaand and H. Çakır, "On continuity of soft mappings," Appl. Math. Inf. Sci. vol. 9, no. 1, pp. 403-409, 2015. 
[7] S. Eilenberg and S. M. Lane, "General theory of naturel equivalences," Transactions of American Mathematical Society, vol. 58, 231-94, 1945.

[8] S. K. Sardar and S. Gupta, "Soft category theory-an introduction," Journal of Hyperstructures, vol. 2, no. 2, pp. 118-135, 2013.

[9] N. Çağman and S. Enginoğlu, "Soft set theory and uni-int decision making," Eur. J. Oper. Res., no. 207, pp. 848-855, 2010.

[10] S. Awoday, Category Theory, Oxford Science Publication, 2010.

[11] T. S. Blyth, Categories, Longman, 1986.

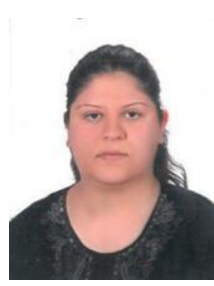

Simge Öztunç was born in Izmir, Turkey in 1982. She received her B.S degree in mathematics from Faculty of Art and Sciences, Celal Bayar University in 2005 and the M.Sc. in topology from Graduate School of Natural and Applied Sciences, Celal Bayar University in 2009. She received her PhD degree in 2013.

Now she is currently working as an assistant prof. doctor at Celal Bayar University, Faculty of Art and
Sciences, Department of Mathematics. She is interested in algebraic topology, digital topology, general topology, fixed point theory and functional analysis. She has five published articles in the international journals. Her fourteen papers have been presented in national and international conferences. 